\title{
TORSION OF OVARIAN CYST DURING PREGNANCY (LAPAROSCOPIC CYSTECTOMY): A CASE REPORT
}

\author{
PARVEEN AKHTER SHAMSUNNAHAR ${ }^{1}$, ABDUL MOTALEB $^{2}$, KHAIRUNNAHAR $^{3}$, NURJAHAN BEGUM $^{4}$, \\ TARAFDER RUNA LAILA ${ }^{5}$
}

\begin{abstract}
In this case we report a 27 year old primigravida having 14 weeks of pregnancy with torsion of ovarian cyst. She presented to a private clinic with acute pain in right lower abdomen. She was diagnosed to have torsion of ovarian cyst during pregnancy and laparoscopic cystectomy was done. Histopathology report showed benign serous cystadenoma. Her pregnancy was followed up. She delivered a healthy baby at term. Though the safety of antepartum surgical intervention has been accepted, laparoscopic surgery needs more advanced surgical skill and knowledge.
\end{abstract}

Key Words: Pregnancy, Ovarian cyst, Laparoscopic cystectomy,

\section{Case Presentation:}

27 years old primigravida attended a private clinic at 14 weeks of pregnancy with acute onset of severe pain in right lower abdomen. The pain was continuous and non radiating in type. It was associated with several episodes of vomiting. She gave no history of vaginal bleeding or discharge. There was no history of diarrhoea, constipation,

1. Dr. Parveen Akhter Shamsunnahar, Assistant Professor, Dept. of Obs. \& Gynae, BSMMU, Dhaka

2. Dr. Abdul Motaleb, Associate Professor, Department of Medicinem, Shahid Ziaur Rahman Medical College, Bogra

3. Dr. Khairunnahar, Associate Professor, Dept. of Obs. \& Gynae, BSMMU, Dhaka

4. Dr. Nurjahan Begum, Assistant Professor, Dept. of Obs. \& Gynae, BSMMU, Dhaka

5. Dr. Tarafder Runa Laila, Assistant Professor, Dept. of Obs. \& Gynae, BSMMU, Dhaka

Correspondence to : Dr. Parveen Akhter Shamsunnahar, Assistant Professor, Dept. of Obs. \& Gynae, BSMMU, Dhaka, E-mail: drsurovi634@gmail.com fever, urinary complaints or any recent illness. She conceived after ovulation induction. She had regular antenatal check up.

No significant past medical and surgical history noted.

On general examination, she was mild anaemic,, blood pressure was 100/70mm of $\mathrm{Hg}$ and a pulse of 88 beats /min. Abdominal examination revealed a palpable tender mass in right lower abdomen to the level of the umbilicus. Pelvic examination revealed a 16 weeks sized uterus, with closed cervix and a tender $10 \mathrm{~cm}$ right adenexal mass.

Her all blood and urine investigations were within normal limit. Transabdominal Ultrasonography revealed $10 \times 6 \mathrm{~cm}$ simple cyst arising from right ovary. Color Doppler flow showed normal vascularity. The left ovary appeared normal and no free fluid was seen in culdesac. There was single intrauterine live fetus of 14 weeks size.

With a provisional diagnosis of ovarian torsion , decision of laparoscopy was taken. $1^{\text {st }}$ port was given in modified palmer position (mid point between tip of left ninth costal cartilage and umbilicus) as the mass extended upto umbilicus and other ports were given in usual way.

On entry into abdominal cavity a congested $12 \mathrm{~cm}$ right ovary was found. It was twisted around its ovarian pedicle 2.5 rotations. . After untwisting ovary returned to its normal color and showed no signs of hemorrhage or necrosis. It contained a $12 \mathrm{~cm}$ simple cyst. Cystectomy was done laparoscopically. The cyst was punctured and the material was sucked out. Then 
it was brought out by $10 \mathrm{~mm} 1^{\text {st }}$ port. Histopathologically it was diagnosed serous cystadenoma of ovary.

Patient recovered without any problem. Tocolytics was given. She was discharged on $3^{\text {nd }}$ postoperative day. The remainder of her pregnancy was uneventful and she delivered an alive male baby by caesarean section at term.

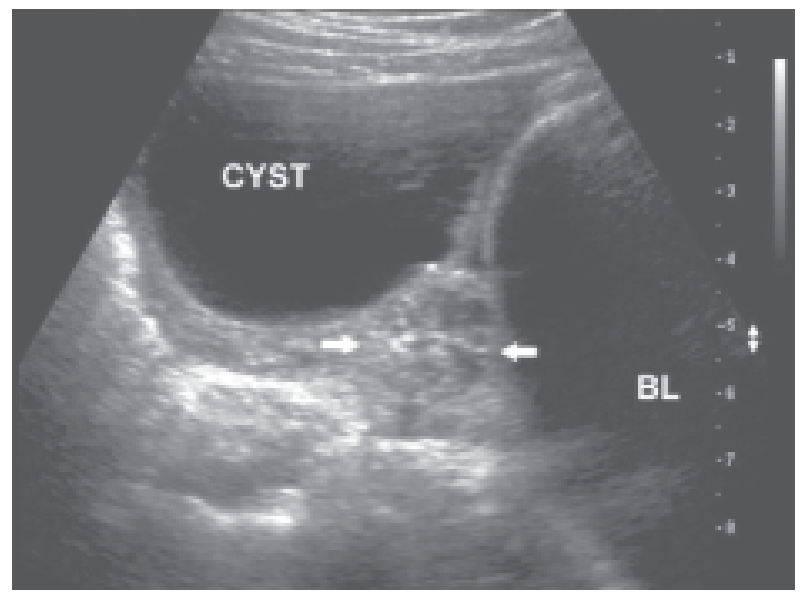

USG of Ovarian cyst

\section{Discussion:}

Ovarian torsion is more common during pregnancy than nonpregnant state. It is associated with severe pain and often difficult to diagnose clinically.

\section{Etiology and Pathophysiology:}

Torsion of the ovary is the total or partial rotation of the adenex around its vascular axis or pedicle. Moderate size, free motility and long pedicle are predisposing factors. The exact etiology is unknown.

Venous or lymphatic blockade could result in potentially massive enlargement of the ovary caused by continued arterial inflow without venous outflow. Eventually if undiagnosed and untreated, arterial stasis can lead to hemorrhagic infraction and necrosis of the ovarian stroma.

The incidence of ovarian torsion rises by 5 fold during pregnancy. Incidence is 5 per 10,000 pregnancies $^{1}$. Its most common cause in pregnancy is a corpus luterm cyst which usually regresses spontaneously by the second trimester ${ }^{2}$. Ovarian torsion, therefore, occurs most frequently in the first trimester, occasionally in the second, and rarely in the third. ${ }^{3}$

Like our patient, ovarian torsion occurs more often in the right adnexae, presumably because the sigmoid colon limits the mobility of the left ovary. ${ }^{4}$ Torsion usually occurs, when the ovary is enlarged secondary to cysts or neoplasms. The majority of cysts are functional. The most frequent nonfunctional neoplasms are serous or mucinous cystadenomas, benign cystic teratomas (dermoids) and ovarian fibroimas. Malignant tumors occur in less the $6 \%$ cases $^{5}$.

Serous cysradenoma, as in our patient, are relatively common accounting for approximately $8 \%$ of ovarian neoplasms ${ }^{6}$.

Serous cystadenomas are usually thin walled, transluscent cysts. Majority are unilocular .Few may have daughters cysts. They are often unilateral, can be bilateral. $10-15 \%$ of them are borderline malignant while $20-40 \%$ are malignant.

Differential diagnosis includes: uterine leiomyomas, non pregnant horn of bicornuate uterus, appendiceal abscess, diverticular abscess, pelvic kidney, retroperitoneal tumours, ectopic pregnancy and retroverted gravid uterus?

\section{Clinical manifestations and diagnosis}

Ovarian torsion can sometimes be difficult to diagnose in pregnancy. The most common clinical presentation is acute onset of severe, colicky unilateral pelvic pain that is usually continous but can wax and wane in cases of incomplete, intermittent torsion. Fall in blood pressure and heart rate is another common response to visceral and deep somatic nociception ${ }^{8}$.

Ultrasound is the diagnostic modality of choice and will most often reveal a unilateral ovarian enlargement that appears solid, cystic, or complex, with or without fluid in the pouch of Douglas. Color Doppler sonography often shows an ovarian enlargement without perfusion of the parenchyma ${ }^{9}$. In the second and third trimester of pregnancy, the ovaries are sometimes difficult to visualize ultrasonographically, because they are displaced form the pelvis by the enlarging uterus. If the ovaries are not clearly visualized with vaginal or abdominal ultrasound, magnetic resonance imaging (MRI) can be used to avoid the risk of ionizing radiation. MRI findings consistent with ovarian torsion include a thick edematous pedicle and ovary, lack of enhancement, and signal intensities with hemorrhage ${ }^{10}$.

\section{Treatment}

Surgery is the treatment for ovarian torsion. The decision to proceed to surgery during pregnancy is 
somewhat complex, since the well-being of both mother and fetus must be taken into account.

The risk of any surgery to the pregnancy will depend on the gestational age. In the first trimester, when ovarian torsion most often occurs in pregnancy, the risk of fetal loss is the smallest with modern anesthetic techniques ${ }^{11}$. Surgery during the second or third trimester is associated with the risk of premature labor. In one study, preterm labor occurred in $26 \%$ of women who had surgery during the second trimester and in $82 \%$ of those who had surgery during the third trimester ${ }^{11}$.

Several approaches can minimize the risk of premature labor. Regional anaesthesia should be used whenever possible to decrease postoperative pain and the subsequent release of catecholamines, which can stimulate uterine contractility. ${ }^{12}$ Continued epidural infusion of narcotics for up to 72 hours in an excellent way to minimize postoperative pain ${ }^{13}$.

Uterine monitoring during surgery is controversial, since fetuses appear to do well as long as the mother is well oxygenated ${ }^{14}$. In a maternal crisis, resuscitation of the mother rather than delivery is the ideal approach. Uterine monitoring in the immediate postaneathesia period for patients in the second or third trimester is an important method for the early detection of regular uterine contractile activity.

Adrenal masses are among the most common indications for surgery during pregnancy. ${ }^{15}$ For years, the treatment of choice for ovarian torsion was salpingooophorectomy, taking special care to avoid untwisting the ovarian pedicle to prevent emboli and toxic substance related to hypoxia from entering the

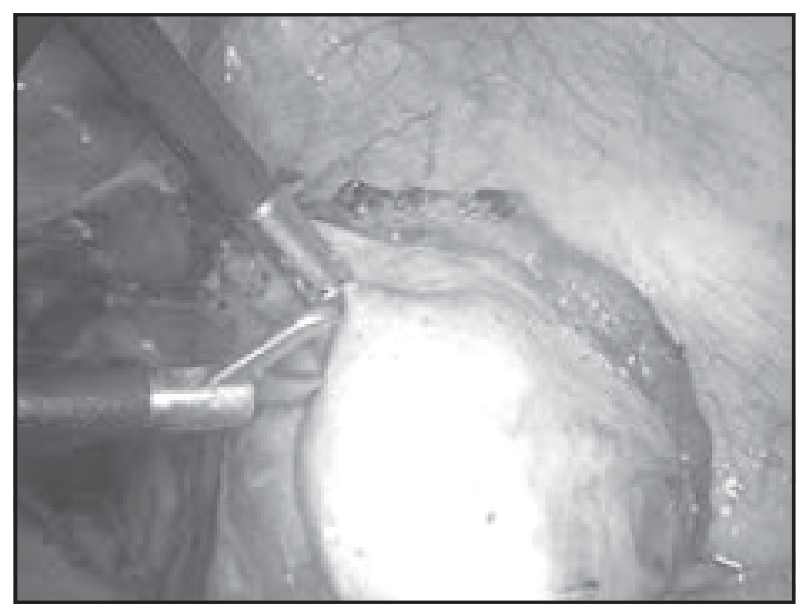

Laparoscopic ovarian cystectomy peripheral circulation. Conservative treatment appears to be warranted to preserve fertility, even for adnexa that initially appear nonviable and purple or black in color $^{16,17}$. However, reestablishing ovarian pedicle has recently been shown to result in viable ovarian tissue on the affected side, with no systemic complications reported to date $11,17,18$.

The main debate is whether the surgery can be performed by laparoscopy or by laparotomy. In the nonpregnant patient and during the first trimester of pregnancy, ovarian torsion can usually be approached laparoscopically ${ }^{15}$. Although untwisting the ovarian pedicle is relatively simple, ovarian cystectomy requires more advanced laparoscopic skill.

During pregnancy due to the physiological changes that take place in the mother and considering the presence of a living fetus in utero, surgical conditions are handled with a lot of care and cautions. In past, pregnancy was considered to be absolutely contraindicated for laparoscopic intervention, but with better understanding of physiology of pregnancy and improved anaesthetic and laparoscopic techniques, emergency laparoscopic procedures such as diagnostic laparoscpy, adnexal surgery, appendicectomy, splencetomy, cholecystectomy and management of ectopic and heterotrophic pregnancies are relatively safe and effective during pregnancy ${ }^{12}$. In 1999, Lachman et al. reported on a series of pregnant women undergoing 518 surgical procedures. Cholecytectomy (45\%) is the most common procedures performed during pregnancy followed by adnexal surgery (34\%) and appendicectomy (15\%). ${ }^{13}$

The responsibility of caring for two patients during one operation and the concern over potential harm to the unborn fetus due to the pneumoperitoneum and / or instrumentation are factors that have played a role in the delay of adapting laparoscopic surgery safe to the pregnant patient. However, recent evidence suggests that not only is laparoscopic surgery safe in the pregnant patient in all three trimester, but it is also often preferable.

With growing ability of minimal access surgeons the recent accumulating data shows that ideal method for commencing pneumoperitoneum is open Hasson trocar method. Tocolysis is indicated if signs of uterine irritability are present. Placement of trocar depends on the size of gravid uterus. Patient can be placed in dorsal lithotomy position in the first - half of pregnancy, but in second-half to prevent inferior vena cava 
compression patient is ideally placed in lateral recumbent position. Operating time should be decreased by using adequate number of ports, and using the most experienced surgeons. Maternal hyperventilation to maintain end-tidal $\mathrm{CO} 2$ pressure at $32 \mathrm{~mm} \mathrm{Hg}$ and lower $\mathrm{CO} 2$ insufflations pressure of $<12 \mathrm{~mm} \mathrm{Hg}$ should be used to avoid fetal acidosis. Electrocautery should be used with care; the smokes containing carbon monoxide should be evacuated promptly to avoid toxic effect to fetus. Entry of all instruments must be under direct vision; care should be taken to avoid injury to the gravid uterus. All specimens should be removed with endobag to avoid spillage. Manipulators should never be fixed to vagina or cervix., Pneumoperitoneum enhances lower extremity venous stasis already present in the gravid patient and pregnancy induces a hypercoagulation state. Therefore, pneumatic compression devices should be utilized whenever possible, Given the enlarged gravid uterus, abdominal access should be attained using an open technique. Dependent positioning should be utilized to shift the uterus away from the inferior vena cava.

There are certain advantages of laparoscopy in pregnancy like short hospital stay, early return to normal activities, small incision, so rapid postoperative recovery and less incision complications such as hernia, postoperative wound infection and pain, less uterine manipulation and hence decrease uterine irritability and fetal loss.

\section{Conclusion}

Ovarian torsion is relatively uncommon in the second trimester of pregnancy. Diagnosis can usually be made on the basis of the characteristic clinical presentation with ultrasound evidence of a unilaterally enlarged adnexal mass. Treatment options are limited to surgery, either by laparoscopy or laparotomy. A laparoscopic access to pathology in pregnancy has many benefits for the patient, but it is important that the surgeon and anesthetist both have an immense knowledge of maternal fetal physiology. An experienced surgeon can continue to perform laparoscopy safely in all trimester by without significant increase in either maternal or fetal morbidity or morality.

\section{References:}

1. Kemmann E, Ghazi DM, Corsan GH. Adnexal torsion in menotropin - induced pregnancies. Obstet Gynaecol 1990; 76: 403-406.

2. Duic Z, Kukura V, Ciglar S, et al. Adnexal masses in pregnancy: a review of eight cases undergoing surgical management. Eur J Gynaecol Oncol 2002; 23: 133-134.

3. Hibbard LT. Adnexal torsion. Am J Obstet Gynaecol 1985; 152: 456-461.

4. Chambers JT, Thiagarajah S, Kitchin JD $3^{\text {rd }}$. Torsion of the normal fallopian tube in pregnancy. Obstet Gynaecol 1979; 54: 487-489.

5. Hess LW, Peaceman A, O'Brien WF, et al. Adnexal mass occurring with intrauterine pregnancy: report of 54 patients requiring laparotomy for definitive management. $A m \mathrm{~J}$ Obstet Gynaecol 1988; 158: 1029-1034.

6. Fatum M, Rojansky N, Shusan A. Papillary serous cystadenofibroma of the ovary? Is it really so rare? Int. J Gynaecol Obstet 2001; 75:85-86.

7. Lee $\mathrm{CH}$, Raman S, Sivanesaratnam V: Torsion of ovarian tumors: a clinicopathological study. Int. J Gynaecol Obstet 1989; 28: 21-25.

8. Cavun S, Goktalay G, Millington WR. The hypotension evoked by visceral nociception is mediated by delta opioid receptors in the periaqueductal gray. Brain Res. 2004; 1019: 237-245.

9. Van Voorhis BJ, Schwaiger J, Syrop CH, et al. Early diagnosis of ovarian torsion by color Doppler sonography. Fertil Steril.1992; 58: 215-217.

10. Zweizig S, Perron J, Grubb D, et al. Conservative management of adnexal torsion. Am J Obstet Gynaecol. 1993; 168: 1791-1795.

11. Visser BC, Glasgow RE, Mulvihill KK, et al. Safety and timing of nonobstetric abdominal surgery in pregnancy. Dig Surg 2001; 18: 409417.

12. Hurd WW, Smith AJ, Gauvin D, et al. Cocaine blocks extraneuronal uptake of norepinephirne by the pregnant human uterus. Obstet gynaecol 1991; 78: 249-253.

13. Jayr C, Beaussier M, Gustafsson U, et al. Continuous epidural infusion of ropivacaine for postoperative analgesia after major abdominal 
surgery: comparative study with i.v. PCA morphine. Br J Anaesth 1998;81:887-892.

14. Horrigan TJ, Villarreal R, Weinstein L. Are obstetrical personnel required for intraoperative fetal monitoring during nonobsterics surgery? J Perinatol 1999; 19:124-126.

15. Chapron C, Capella-Allouc S, Dubuisson JB. Treatment of adnexal torsion using operative laparoscopy. Hum reprod 1996; 11: 998-1003.

16. Cohn SB, Oelsner G, Seidman DS, et al. Laparoscopic detorsion allows sparing of the twisted ischemic adnexa. J Am Assoc Gynaecol Laparosc 1999; 6: 139-143.
17. Lee Ch, Raman S, Sivanesaratnam V. Torsion of ovarian tumors: a clinicopathological study. Int. J Gynaecol Obstet 1989;28:21-25

18. Pan HS, Huang LW, Lee CY, et al. Ovarian pregnancy torsion. Arch Gynaecol Obstet 2004; 270: 119-121.

19. Al-fozan H, Tulandi T. Safety and risks of laparoscopy in pregnancy. Curr Opin Obstet Gynaecol 2002;14: 375-9.

20. Lachman E, et al. Pregnancy and laparoscopic surgery. J Am Assoc Gynaecol Laparosc 1999; 6(3): 347-51. 